A LIVELY ELECTRONIC COMPENDIUM OF RESEARCH, NEWS, RESOURCES, AND OPINION

Astronomy Education Review

Volume 6, Aug 2007 - Mar 2008

Issue 2

\title{
Regulations and Ethical Considerations for Astronomy Education Research II: Resources and Worked Examples
}

\author{
by Erik Brogt \\ The University of Arizona \\ Erin Dokter \\ The University of Arizona \\ Jessie Antonellis \\ The University of Arizona \\ Sanlyn Buxner \\ The University of Arizona \\ Received: 11/05/07, Revised: 01/02/08, Posted: 01/30/08
}

The Astronomy Education Review, Issue 2, Volume 6:99-110, 2008

(C) 2008, Erik Brogt. Copyright assigned to the Association of Universities for Research in Astronomy, Inc.

\begin{abstract}
This article discusses the legal and ethical requirements of human subjects research proposals in astronomy education research. We present an overview of the relevant laws, regulations, and guidelines that inform an Institutional Review Board evaluation of proposed research. We also present examples of potential research projects in astronomy education research and discuss their ethical issues.
\end{abstract}

\section{INTRODUCTION}

Astronomy education research, as a field of sociobehavioral research, generally involves the participation of human subjects. As such, it is regulated by federal law and ethical guidelines to protect the rights of, and minimize risk to, participants. Failure to observe these laws and guidelines can have serious ramifications, both for the individual researcher and his or her institution. In an earlier article on the legal and ethical issues in astronomy education research (Brogt, Dokter, \& Antonellis 2007, hereafter, Paper I), we outlined the general procedures and relevant laws that govern research on human participants in the United States, and the role of the Institutional Review Board (IRB) in all phases of research study approval. In this article, we outline the legal requirements implemented by IRBs with respect to research proposals. Not all institutions have an IRB, but if you partner with an institution that does, you must adhere to the policies of that institution. In all cases, the policies of the institution with the strictest requirements must be followed. We will discuss ethical and legal issues for astronomy education researchers working at institutions without an IRB in an upcoming article. 


\section{ESTABLISHING AN IRB AND BECOMING CERTIFIED (OR, "GETTING STARTED")}

As discussed in Paper I, all astronomy education research in the United States conducted by researchers at institutions with an IRB is governed by federal law and ethical guidelines. By law, institutions receiving federal money to conduct medical or sociobehavioral research must set up an IRB or go through the IRB of a partner institution. The law states that "An institution becomes 'engaged' in human subjects research when its employees or agents (i) intervene or interact with living individuals for research purposes; or (ii) obtain individually identifiable private information for research purposes" (45 CFR 46.102(d)(f), U.S. Dept. of Health and Human Services 2005).

In general,

- If your institution receives federal funding for research with humans, your institution must create an IRB, and research studies must go through the approval process.

- If your institution does not receive federal funding for research with humans, there are no formal regulations that govern your research activities. It is recommended that astronomy education researchers working in these situations follow the ethical principles outlined in the Belmont Report (National Commission for the Protection of Human Subjects of Biomedical and Behavioral Research, 1979). One can also consider going through human subject training (discussed below). We will discuss these options in more detail in an upcoming article.

The Office of Human Research Protections (OHRP) within the United States Department of Health and Human Services maintains a very informative and comprehensive Web site on all the requirements for the establishment of an IRB: http://www.hhs.gov/ohrp. The site contains information on setting up an IRB http://www.hhs.gov/ohrp/assurances/assurances_index.html, contact information for individual officers assigned to each state (http://www.hhs.gov/ohrp/daqi-staff.html\#staff), a Frequently Asked Questions page http://www.hhs.gov/ohrp/faq.html, and the text of the code of federal regulations to which we refer in this article http://www.hhs.gov/ohrp/humansubjects/guidance/45cfr46.htm.

These regulations typically do not apply to NASA and NSF grants that have an education/public outreach (E/PO) component. Note that the phrasing usually refers to an E/PO component, such as NASA outreach related to its missions. $\mathrm{E} / \mathrm{PO}$ or evaluation of $\mathrm{E} / \mathrm{PO}$ is not considered education research. An IRB only reviews research projects, not E/PO projects. However, should you want to conduct research on an E/PO project, publicize the results, or publish your findings or even anecdotal information resulting from the project, it would become a research study and you would have to go through IRB approval, as mentioned in Paper I.

One of the requirements for conducting astronomy education research under an IRB is that the researchers be trained in the ethical considerations of sociobehavioral research, including having an understanding about the IRB process, the regulations for consenting participants, and the rights of study participants. The specific training program used by an institution is determined by the IRB. For example, researchers at the University of Arizona are trained using the Collaborative Institutional Training Initiative (CITI, http://www.citiprogram.org//, a collaboration between the University of Miami and the Fred Hutchinson Cancer Center. The Web site includes an extensive Frequently Asked Questions page to help institutions and individuals get started. 
Once training is complete, you are free to prepare and submit an IRB proposal for the research study that you have in mind. In the proposal, you need to outline what you want to do, why you want to do it, how you are going to do it, and what safeguards you have in place to minimize the risk to your participants. In the next section, we briefly discuss what elements typically go into such a proposal, and the evaluation rubric that the IRB uses to assess your research proposal.

\section{PREPARING AN IRB PROPOSAL}

The requirements for approval of a study are based on the Code of Federal Regulations: Criteria for IRB Approval of Research (45 CFR 46.111); General Requirements of Informed Consent (45 CFR 46.116); and Documentation of Informed Consent (45 CFR 46.117). Based on these broad federal guidelines, individual IRBs formulate specific policies, which may also include items required by accrediting agencies or other professional bodies. In general, the IRB will want detailed information about the participants, recruitment and consenting procedures, data collection processes, confidentiality of data, and benefits and risks. As an example, we highlight the sections of the Project Review Form used by the Human Subjects Protection Program (HSPP) at the University of Arizona, a Research I institution that engages in both biomedical and sociobehavioral research.

\section{General information}

To begin your proposal, you will need to describe your project, summarizing the background, goals, methods, and significance. This is your first opportunity to explain what you will be doing and why. Other general information solicited by the HSPP includes the name of the individual(s) who will be the principal investigator(s) of the project and others who will be involved with consenting participants. So, for instance, if you have a graduate assistant who would be introducing participants to the project and obtaining consent from them, his or her name would need to be listed on the proposal. All study personnel must receive training in human subjects research through CITI or whatever agency your institution's IRB stipulates.

The IRB will also inquire into the duration of the project, including the date that the study will begin (when you are planning to start recruiting participants) and the date that it will be complete (this includes completion of data analysis). Make sure you set dates carefully to allow yourself enough time to complete the project. Check with your institutional HSPP to find out how far in advance you will need to submit your proposal to allow sufficient time for a complete review prior to the initiation of data collection. Your HSPP may require yearly resubmissions for the duration of your project (however, exempt projects do not always require annual review, though some institutions require it if minors or vulnerable populations are involved). Participant recruitment and data collection may not begin until IRB approval is granted.

\section{Population}

In describing your population, report whom you will be recruiting (including age range, gender, and ethnicity, if appropriate), why you will be recruiting from this population (for instance, you might be recruiting participants from among the subscribers to an astronomy education e-mail list (LISTSERV) because you're interested in the experiences of astronomy educators), and an estimate of how many people will be invited to participate (e.g., there are 200 subscribers to the e-mail list, and you would like to interview 25 individuals). Note that you will need to seek a modification to your protocol should you want to increase your sample size beyond the limits you specified. You may be asked to report any special 
efforts to encourage the participation of certain groups (e.g., women or minorities). Other considerations will be how you are selecting participants (e.g., will you be choosing individuals at random from among those who agree to participate, or will you seek a balanced representation by gender and ethnicity?).

You will also need to report whether your recruitment pool may include members of any vulnerable populations. When determining whether your population would be regarded as vulnerable by an IRB, consider if there may be any reason to believe your participants are especially vulnerable to coercion and/or exploitation. The federal regulations provide a list of vulnerable populations, though it is clear from the wording that the list is not meant to be exhaustive (45 CFR 46.107a). Children are an example of a vulnerable population; as minors, they are not legally able to provide consent on their own behalf, but rather to assent. You must solicit permission from a parent or legal guardian to include a minor in research, and seek the assent (an affirmative agreement to participate in research) of the minor. Whether assent is needed is determined by the IRB, which looks at the age, maturity level, and psychological state of the minors involved (45 CFR 46.408a). Other vulnerable populations mentioned in the federal regulations are pregnant women, prisoners, and the cognitively impaired. Examples of vulnerable populations not mentioned explicitly in the federal regulations are students, employees, non-English speakers, the elderly, tribal members, individuals in a low economic bracket, and individuals who may be at risk as a result of sharing information with you because of work or political conditions.

Last, you will need to discuss the inclusion and exclusion criteria for potential participants. For instance, you may want to recruit participants from all students enrolled in an Astronomy 101 class but exclude any minors, who cannot legally give consent (remember that college classes often include students under the age of 18).

\section{Recruitment and consenting procedure}

In this section, you will describe your recruitment process and the way in which the study will be explained to participants. For instance, you may be using phone calls, flyers, e-mail, or a television commercial. Describe both the recruitment methods you will be using and the specifics of what your recruitment will entail (e.g., the text of e-mails or scripts for phone calls). The human protection office will want to know what you plan to tell potential participants about the project. Refer to any recruitment documents, which should be included in your proposal.

You will also need to describe the process by which an individual becomes a participant. For example, will you put your e-mail address on your recruitment materials so that potential participants can contact you? Or are you planning to contact potential participants individually via e-mail? The IRB will be looking to see that your methods are ethical and protect your participants from unnecessary risk (see Section 3.1). Avoid public sign-up sheets or other means of recruiting by which individuals volunteering for a study could be identified, because this compromises participant confidentiality. Only the study personnel should know who has volunteered to participate in the study.

Next, discuss how participants will provide their consent. There are several ways to do this. For instance, the principal investigator or others involved with the research may sit down privately with each participant and read through the consenting document with him or her, ensuring that each participant is aware of the details of the project and providing the opportunity for the participant to ask questions before signing the consent documents. Alternatively, you might allow participants to take the consenting documents with them and think them over until a later time. Written consent may not be possible or necessary for your study; for instance, you might use an electronic survey and allow individuals the option of participating in 
the study electronically. Or, depending on the nature of the study, you can provide a disclaimer on a survey stating that submitting the survey implies consent to use the data for research purposes. In some cases, participants can provide oral consent. Check with your individual human protection office for guidance.

All written and oral statements shared with your participants need to be reviewed and approved by the IRB. The IRB will be looking for the possibility of manipulation or coercion of potential participants. For instance, participants can be misled by virtue of their being unable to comprehend your study or read your documents (as may be the case with the cognitively impaired, non-English speakers, children, and possibly the elderly). To avoid this possibility, compose your consenting and recruiting documents in nontechnical language that clearly states the nature of the study and what you're asking of participants. Have your documents translated if need be. Individual IRBs may set additional requirements on the translation-for example, to have the translation certified by a native speaker of the language. If you'll be including minors in the study, explain how you will disseminate information to, and obtain consent from, the minors' parent or guardian. Include language in your recruitment and consenting documents that communicates that participants may withdraw from the study at any time, without retribution, and that their participation is voluntary. It is especially important that care is taken to inform participants of their rights when there is a power differential between the researcher(s) and participants, such as when the participants are students in the researcher's class.

\section{Methodology and data collection procedures}

Here you will elaborate fully on the methods and procedures that you intend to use for your data collection. The IRB will want to know the specific nature of the data that you will be collecting (e.g., if you intend to use questionnaires or surveys, make observations, videotape or audiotape participants, take photographs, conduct interviews or focus groups, review documents, or use any other method). It is also customary to include a copy of your intended instruments in your proposal (e.g., surveys, interview questions, or observation protocols). If you must obtain written approval from other entities to collect your data, these approvals should be attached as well. This may include authorization from a designated official to collect data at a location other than your own institution. If you are engaging in a collaboration with researchers at other institutions, include your plans for data collection at the different sites.

Also include in this section the procedures for protecting participants' privacy while they are involved with the study. Consider, for instance, whether you can conduct individual interviews rather than focus groups to provide a higher level of privacy, or conduct interview/focus groups away from the participants' places of employment, schools, or residences. Whenever possible, data collection should take place in a location mutually agreed on by investigators and participants to make the participants feel more comfortable. The location should minimize the participants' risk should there be any.

\section{Confidentiality of personal information}

This section of your proposal involves descriptions of how researchers will ensure that data collected from participants are kept confidential. This includes providing detailed information regarding how the data will be stored and who will have access to it, and how data will be separated from any personally identifiable information about participants. Researchers are responsible for ensuring that participants' confidentiality is protected. Be aware of who has access to the computers on which data is stored, who will handle data that can be linked to specific individuals, and whether links between data and participants (e.g., a list of participants' real names, code numbers, or pseudonyms) will be destroyed when the study is complete. 
Benefits, costs, compensation, and risks

Here you will describe any advantages and disadvantages of participation in the project, which should be outlined in your consenting documents as well. Can participants expect any direct benefits from being involved in the study? For instance, participants may benefit from participating in a study in which they interact with peers to discuss a topic about which they are learning, because the experience allows them to gain a deeper understanding of this topic. Are there any broader benefits of participating in the study, such as contributing to the improvement of museum offerings? What are the costs to participants? There will always be some cost, usually in the form of time in the case of astronomy education research.

Benefits from participation are distinct from compensation, which refers to money or other rewards offered in gratitude for participation in the study. Compensation can be unduly coercive if care is not taken; for instance, if you offer $\$ 500$ compensation for a half-hour's participation to individuals in a low economic bracket, it would be virtually impossible for them to say no, and you will have compromised their ability to decline to participate. Be sure to offer compensation that is commensurate with what you are asking of participants.

Typically, astronomy education research will not pose physical risks to participants, but you must consider and describe any other potential risks, such as psychological risk. If you are collecting data from your own students, they may feel anxious about looking stupid in front of their professor or be concerned that their grades will be affected if they do or do not participate in the research. If you are collecting data from visitors to a museum, they may be uncomfortable with feeling like they're being watched. If you are collecting data from your colleagues, they may experience stress related to sharing information that could compromise their professional status. Be mindful of how your participants could be harmed, and outline ways that you can eliminate or mitigate these risks. For instance, what will you tell participants to assure them that their data will remain confidential, not be used against them, not be shared with their supervisors, and so on?

\subsection{Evaluation Rubric}

The proposal will be evaluated by the IRB using an evaluation rubric. Different institutions may have different rubrics. At the University of Arizona, the rubric examines risk/benefit, subject selection, consent, monitoring of data, and treatment of vulnerable populations. Each item is scored as "Yes," "No," or "N/A." We present the abbreviated rubric below, leaving out the elements that are geared toward medical research because those are not relevant for $A E R$.

Risk

- Are risks to participants minimized by using procedures that are consistent with sound research design and that do not unnecessarily expose subjects to risk?

- If yes to the above question, are risks to participants minimized by using those procedures?

- Are risks to participants reasonable in relation to anticipated benefits to participants (if any), and the importance of the knowledge that may be expected to result?

\section{Subject selection}


- Is selection of participants equitable?

Consent

- Will the investigator obtain the legally effective informed consent from the participant or the participant's legally authorized representative (i.e., is the participant able to understand the consent form)?

- Will the informed consent be appropriately documented?

\section{Monitoring of data}

- When appropriate, will the research plan make adequate provision for monitoring the data collected to ensure the safety of subjects?

- When appropriate, are there adequate provisions to protect the privacy of subjects and to maintain the confidentiality of data?

\section{Vulnerable populations}

- When some subjects are likely to be vulnerable to coercion or undue influence, such as children, prisoners, pregnant women, mentally disabled people, and economically or educationally disadvantaged persons, have additional safeguards been included in the study to protect the rights and welfare of these participants?

When the IRB members have completed their evaluation of your proposal, they will notify you as to whether your project is approved, whether clarification is needed, or what changes must be made to the research protocol to obtain approval. As soon as your protocol is approved, you can start recruiting participants and collecting data.

\section{EXAMPLES}

In this section, we provide a few working examples of research studies, the likely response that they would receive from an IRB, and how a researcher could mitigate these issues. It should be noted that the IRB only reviews the legal issues and ethical considerations associated with the study. In the following examples, we leave it to the reader to judge the quality of the research study examples.

\subsection{Example 1: Using Exam Scores for Research}

\section{The idea}

"I want to use the final exam scores from my two different classes to see which instructional strategy is more effective. I decided to use only lecture with my first class and added a learner-centered strategy of think-pair-share to my second class in addition to lecture. I want to give students who participate some extra credit on their final exam."

\section{Potential ethical pitfalls}


- Nonvoluntary participation: Everyone has to do the final or his or her course grade will be substantially lower, and this can be considered coercion for participation. You do not have clear procedures for opting out: students are not given the opportunity to indicate whether they give consent for their exams to be used for research purposes.

- Withholding treatment: You are withholding a potentially beneficial teaching strategy from one class for no pedagogical reason. Withholding it can be detrimental to one class's performance.

- Inappropriate compensation: Giving extra credit to students participating in the study is coercive; students may perceive that their grades will suffer if they do not participate.

\section{Potential solution}

Students should be given a statement that indicates what the research is about and what the data will be used for. They should have the opportunity to decline to have their exam included in the study without fear of retribution or fear that their grade is at stake; the statement should assure students that their participation is voluntary and entirely separate from any evaluation of their performance in the class. One solution is to give all students who came to class that day extra credit, regardless of participation in the study.

Withholding an effective instructional strategy from one class should raise a red flag with your human protection office because it impacts students' learning experiences. Remember that you are an educator first, and your obligation is to your students before your research. The question you should ask yourself is, Why are you repeating a well-documented intervention? You may consider instead using two different learner-centered instructional strategies and comparing their effectiveness for student performance on the final exam. However, this is more a research design issue than an ethical one.

\subsection{Example 2: Using In-Class Survey Results for Research}

The idea

"I am interested in investigating the connection between student achievement and student demographics. I want to hand out a survey for my research in class, where I will ask about students' backgrounds, including ethnicity, family income, and religion. If students fill it out, I will give them participation credit; if they choose not to do the survey, they do not get credit for that day."

\section{Potential ethical pitfalls}

- High-risk research: You are asking for very personal information. Students may not feel comfortable revealing the information, especially if it is not clear what you are going to do with the data. It may also expose them to tokenism, where the students feel cast into the role of being a representative for their demographic, or stereotype threat, in which students' achievement is influenced based on perceived societal stereotypes of their gender, ethnicity, and so on.

- Penalizing nonparticipation: If the participation credit is a regular part of the grade, then students are penalized for choosing not to participate.

- Coercion to participate: The inherent power differential between the students and the instructor, who is also the researcher, can lead students to feel pressured to participate because they can see participation as a class assignment, not something that is voluntary and that they can opt out of. 


\section{Potential solution}

Make very clear why you are asking the students to provide the demographic information and what you are going to do with it. Make sure the class understands that participation in the survey is voluntary. The decision to give or withhold participation credit should be entirely distinct from students' participation in the survey. Making your survey anonymous will further reduce the risk and the pressure to participate.

In practice, you can, for example, do the following: Everyone present in your class that day, whether they participate or not, will, and must, get participation credit. If your survey is anonymous, after collecting the surveys, you can ask all your students to write down their name and student ID on a piece of paper, and collect those to give credit to all students present.

\subsection{Example 3: Control Group Blues}

\section{The idea}

"I am planning a research study on students' knowledge of the night sky. Participation in the study is voluntary. If students want to participate, they can go to a star party in a local science museum half an hour from campus, outside the city. Later in the semester, I will give a constellation quiz to all students as part of the class. I will use the data from the students who did the extra credit opportunity as my treatment group, and the others as the control group."

\section{Potential ethical pitfalls}

- Deception: Potential participants were not fully informed of the nature of the study. Not knowing that they'd be quizzed on it later, students may have opted not to participate, which could hurt their grade.

- Equitability of participant selection: Students without a car or with an evening job are less likely to be able to participate.

- Withholding beneficial treatment: Attending a star party arguably gives participants a greater chance of obtaining a high score on the constellation quiz. The grades of those who did not have the opportunity to participate will suffer as a result.

- Use of nonparticipant data: Being in a control group means being part of the study. If students decline to participate, their data cannot be used at all for research purposes, thus their scores cannot be used as a control.

\section{Potential solution}

A solution would be to have the constellation quiz be non-credit-carrying and to recruit a pool of participants that includes students who did (treatment group) and didn't (the control group) go to the star party, all of whom consent to the use of their data for research purposes.

\subsection{Example 4: Using Data Obtained From Minors for Research}

The idea 
"I want to give fifth graders in our local elementary school a survey to see what they know about black holes. My neighbor, who is the teacher in that class, will send a letter to the parents asking for consent and encouraging them to have their children participate. She will also talk to the students about the survey, administer it, and give me the results."

\section{Potential ethical pitfalls:}

- Site authorization: The district, school principal, and possibly each teacher may have to give permission to use the school and/or classroom as a research site.

- Nonresearch personnel: Recruitment, consent, and assent are done by someone who is not part of the research team and may not be certified to do research on human subjects by your IRB.

- Protection of minors: The teacher has considerable access to data from minors, which may be unlawfully invoked in the course of data collection. The children should be protected because they are a vulnerable population.

\section{Potential solution}

Check both your own institution's requirements and the district's requirements prior to recruiting to see if you must obtain site authorization. School districts often have strict regulations about research conducted within schools. You will need authorization from the principal to enter the school for any research and, most likely, the class teacher's permission to enter the classroom. Note that you do not have to seek the consent of the teacher or principal; they are not part of your study. To obtain consent from participants, the researcher, not the teacher, should send a letter to the parents outlining the research study and the plans for the data. You will also need to secure assent from the students. The teacher should be certified by your IRB to do research if he or she is part of the consenting process or involved in administering the survey, collecting results, and so on. If not, you should seek consent from participants and collect data yourself.

\subsection{Example 5: General Public E/PO}

\section{The idea}

"I am a university researcher, and I want to observe visitors to a local science museum during their visit to a special exhibit. I plan to videotape families during their interaction with an exhibit to determine if they spend more time reading the text panels or working with the interactive exhibit components, and to audiotape their conversations during the time they spend at the exhibit. I do not want to bias their experience by telling them they are being watched, but rather would like to observe them in the most natural setting possible."

\section{Potential ethical pitfalls}

- Lack of informed consent: You have failed to obtain consent for participation, including consent to use data from minors, and the assent of the minors if they are seven years of age or older.

- Site authorization: Without obtaining approval to conduct research at a private institution, you run the risk of violating the institution's policies concerning visitor rights and protections.

- Videotaping/audiotaping without consent: The use of videotaping and audiotaping is considered high risk because you have a permanent record of participants' images and spoken conversations. Videotaping and audiotaping without consent are especially egregious violations of participants' 
rights.

\section{Potential solution}

You may be able to forgo consent if you can make the case that you are observing people in a public, natural setting. However, if you find that audiotaping and videotaping are essential to your research, you must get permission. A possible solution is to approach families at the beginning of their museum visit and ask them to wear microphones for their stay in the museum, obtaining permission to tape anything they say and/or do on the exhibit floor. In this way, you are not emphasizing any specific event in the museum, and you have a better chance of seeing more natural interactions.

Another solution is to not use videotaping or audiotaping, but rather to take field notes and make observations. This makes the observations anonymous because there is no record of identifiable information (pictures, voices). However, even with this solution, you may still have potential issues with site authorization. Many institutions require consenting documents that disclose the purpose and methods of your research.

\section{Conclusions}

In this article, we outlined the steps from becoming a researcher in the sociobehavioral sciences, of which astronomy education research is one, to the details of what an IRB expects your proposal to contain. You begin by becoming certified through a training program, submitting your proposal, and working with the IRB to address any ethical issues that they may have in order to obtain their approval. Only after approval can you start recruiting participants and collecting data. The IRB approval process is in place to ensure that studies involving humans are conducted in an ethical manner that respects the rights of participants. The process protects the researcher (and the institution) if legal and ethical issues are raised about the study. Without this approval from your institution, you may unknowingly be causing harm and leaving yourself open to legal action.

This article was written for researchers working at institutions that have an IRB in place, and we realize that not all institutions have an IRB. In an upcoming article, we will discuss the legal and ethical issues for researchers at those institutions who want to engage in astronomy education research.

We did not intend to address issues of research design in this article. As stated previously, the IRB evaluates legal and ethical issues, including those issues that affect the design of the research. Just as you would not submit an observing proposal for a 4-meter class telescope to go observe the full Moon, your research questions and methods should be fruitful. This argues for a decent grounding in the literature for astronomy education researchers in order to gain an understanding of which questions are worth pursuing and which have already been answered.

\section{Acknowledgments}

The authors wish to thank Dr. Rebecca Dahl, director of the University of Arizona's Human Subjects Protection Program, for her helpful suggestions and review of earlier drafts of this article. 


\section{References}

Brogt, E., Dokter, E., \& Antonellis, J. 2007, "Regulations and Ethical Considerations for Astronomy Education Research," Astronomy Education Review, 6(1), 43. http://aer.noao.edu/cgi-bin/article.pl?id=242.

National Commission for the Protection of Human Subjects of Biomedical and Behavioral Research. 1979, "The Belmont Report: Ethical Principles and Guidelines for the Protection of Human Subjects of Research,"Retrieved April 18, 2007, from http://www.hhs.gov/ohrp/humansubjects/guidance/belmont.htm.

U.S. Department of Health \& Human Services. 2005, Code of Federal Regulations, Title 45: Public Welfare, Part 46: Protection of Human Subjects. Retrieved April 18, 2007, from http://www.hhs.gov/ohrp/humansubjects/guidance/45cfr46.htm.

FR

99 - 110 